\title{
MÉXICO: AVATARES EN EL FINANCIAMIENTO A LA CIENCIA Y LA TECNOLOGÍA ${ }^{1}$
}

MEXICO: AVATARS IN THE FINANCING SCIENCE AND TECHNOLOGY

Heidi Mariana Archundia Esquivel

Correspondencia: marhey04@hotmail.com

\section{RESUMEN}

La investigación científica y tecnológica, en México, es presentada, en discursos de los organismos internacionales, en las políticas nacionales, y hasta en las declaraciones de científicos mexicanos, como el factor fundamental para lograr desarrollo y bienestar nacional, sin embargo, esa valoración de la ciencia y la tecnología no se corresponde con las asignaciones presupuestales que se confieren para promoción de la misma, aunque sí es claro cómo el tipo de actividades que se han organizado para promover la investigación científica y tecnológica se corresponden con determinadas concepciones acerca de lo que es investigación y la caracterización de su estado en el país. Para dar cuenta de ello se examina, en el periodo 1980-2019, el modo en que el financiamiento ha transitado de ciertas actividades de apoyo, proyectos de investigación, etcétera, advirtiendo cómo la evolución del financiamiento, está en relación a la manera en que se concibe el conocimiento científico y tecnológico y su lugar en la educación superior.

$1 \quad$ Este artículo fue asesorado y revisado por el M. en S. Maximiliano Valle Cruz, del Cuerpo Académico de Educación y Políticas Educativas de la Universidad Autónoma del Estado de México. 
Palabras clave: Financiamiento, Investigación Científica y Tecnológica, Actividades de Apoyo, Políticas Públicas Nacionales, Organismos Internacionales.

\section{ABSTRACT}

Scientific and technological research in Mexico is presented, in speeches by international organizations, in national policies and even in statements by Mexican scientists, as the fundamental factor in achieving national development and well-being. However, this evaluation of science and Technology does not correspond to the budgetary allocations that are granted for its promotion, although it is clear how the type of activities that have been organized to promote scientific and technological research correspond to certain conceptions of what research is and the characterization of its state in the country. To explain this, we examined, in the period 1980-2019, the way in which financing has moved from certain support activities, research projects, etc., noting how the evolution of financing is related to the way in which Scientific and technological knowledge is conceived and its place in higher education.

Key words: Financing, Scientific and Technological Investigation, Support Activities, National Public Policies, International Organizations.

\section{INTRODUCCIÓN}

La investigación científica y tecnológica, en las últimas décadas, se ha señalado como fundamental debido a que, continuamente, se incorpora en los procesos económicos, así como en la participación ciudadana en su relación con las funciones del gobierno, hasta tocar la vida cotidiana de las personas. Asimismo, es notorio el modo en que se ha enfatizado, en la actualidad, un discurso que sostiene que desarrollo científico-tecnológico 
e innovación son fundamentales para la prosperidad económica, social y cultural de los países en desarrollo. Esas ideas son elaboradas y difundidas por los organismos internacionales, como ocurre en Los objetivos del desarrollo sostenible, formulados por Naciones Unidas en 2015.

Las concepciones en torno a la investigación científica y tecnológica se instalan en las políticas nacionales, y hasta en las declaraciones de científicos mexicanos, como el factor fundamental para lograr desarrollo y bienestar nacional, sin embargo, esa valoración de la ciencia y la tecnología no se corresponde con las asignaciones presupuestales que se confieren para promoción de la misma, y se concreta en determinadas actividades para promover la investigación que se corresponden con determinadas concepciones acerca de lo que es investigación y la caracterización de su estado en el país. Para dar cuenta de ello se examina, en el periodo 1980-2019, el modo en que el financiamiento ha transitado de ciertas actividades de apoyo, proyectos de investigación, etcétera, advirtiendo cómo la evolución del financiamiento, está en relación a la manera en que se concibe el conocimiento científico y tecnológico y su lugar en la educación superior.

Para cumplir el propósito este artículo se organiza en tres apartados: en el primero se exponen los planteamientos de tres organismos internacionales: la Organización de las Naciones Unidas para la Educación, la Ciencia y la Cultura (UNESCO), la Organización para la Cooperación y el Desarrollo Económicos (OCDE) y la Comisión Económica para América Latina y el Caribe (CEPAL); en el segundo apartado se examinan las políticas públicas mexicanas referidas a la investigación científica y tecnológica y, en el tercero se muestra cómo esas recomendaciones y políticas se han manifestado en cambios en el financiamiento a la educación superior, donde se incluye la investigación. 


\section{MÉTODO}

El objetivo, en el presente artículo, es mostrar cómo las variaciones en el financiamiento a la investigación en ciencia y tecnología en México, desde la década de 1980 al 2019, ha dependido del modo en que se concibe la investigación científica y las estrategias para su desarrollo, lo cual está ligado a las recomendaciones internacionales en torno al financiamiento a la educación superior pública, las que han servido de base para diseñar las estrategias para organizar y promover la investigación científica a escala nacional, tales como becas de posgrado, creación de infraestructura o estímulos al personal, entre otras.

El presente estudio supone una investigación de carácter documentalinterpretativo en la medida que se apoya en la consulta de documentos oficiales de organismos internacionales, así como de políticas educativas nacionales -donde se incluyen datos estadísticos-, asimismo incorpora resultados de estudios realizados por investigadores, poniendo atención a la manera en conceptualizar la investigación científica y tecnológica en distintos períodos, para dar cuenta como se ha modificado dicha concepción, y la manera de considerar cuál es el estado de su desarrollo en países como México, lo cual implica realizar un proceso de interpretación del significado que se concreta en acciones específicas entre 1980 a 2019.

Así, al incorporar la interpretación se trata de destacar los significados que ha tenido la investigación y su desarrollo y cómo se expresa en los cambios en el financiamiento. 


\section{Los organismos internacionales $y$ el financiamiento a ciencia $y$ tecnología}

De acuerdo con la Comisión de Ciencia y Tecnología para el Desarrollo de la ONU (UNCTAD por sus siglas en inglés) el desarrollo de la ciencia y la tecnología se concebía, desde 1949, dentro de la cooperación internacional para "contribuir al desarrollo económico [según] la Conferencia Científica de las Naciones Unidas para la conservación y utilización de los recursos naturales" (Comisión de Ciencia y Tecnología para el DesarrolloUNCTAD,1997:5). Posteriormente, en 1963, Naciones Unidas realizó una conferencia internacional donde estableció que:

...para estudiar las posibilidades de aplicación de la ciencia y la tecnología en beneficio de las regiones menos desarrolladas [para] mostrar los medios que existían para acelerar el desarrollo mediante la aplicación de los avances más recientes en el ámbito de la ciencia y la tecnología [a través de] un esfuerzo renovado y sostenido para facilitar la transferencia de ciencia y tecnología a los países en desarrollo y para ayudarlos a superar los obstáculos que se oponían a su acceso a los conocimientos y a la aplicación de éstos. (UNCTAD, 1997:5-6).

La idea difundida es que el progreso tecnológico era igual a desarrollo, pero era un progreso tecnológico que iba de los países desarrollados a los menos desarrollados, lo cual fue la clara justificación de las inversiones extranjeras directas: la instalación de empresas transnacionales; hasta que, en 1971, Naciones Unidas planteó:

...la necesidad de crear una capacidad autóctona, [reconociendo] la brecha entre las posibilidades de la ciencia y la tecnología y la medida en que se aprovechaban efectivamente, la debilidad de las instituciones científicas en el mundo en desarrollo, el problema del acceso a la tecnología mundial, y el éxodo intelectual. (UNCTAD, 
De acuerdo con el documento de la UNCTAD, los países menos desarrollados solicitaban incrementar el acceso al acervo mundial de ciencia y tecnología, en la década de 1970, de tal modo que, en 1979, Naciones Unidas adoptó un Programa de Acción que comprendía:

i) fortalecimiento de la capacidad científica y tecnológica de los países en desarrollo; ii) reestructuración del sistema actual de relaciones científicas y tecnológicas internacionales en la transferencia de tecnología, y iii) fortalecimiento de la función que corresponde al sistema de las Naciones Unidas en la promoción de nuevas formas de cooperación tecnológica, así como en lo tocante a un aumento considerable de la aportación de recursos financieros para esos fines (UNCTAD,

1997:6).

Vemos pues que, conforme a Naciones Unidas, la ciencia y la tecnología es una posesión de las naciones industrializadas, pues ellas son las desarrolladas que, en un primer momento brindan cooperación para que los menos desarrollados accedan al crecimiento económico; en un segundo momento se plantea en términos de transferencia de ciencia y tecnología hacia los países menos desarrollados; posteriormente se plantea que los países con menos progreso requieren desarrollar capacidades para la investigación científica, tanto en recursos humanos como institucional, mediante aportaciones financieras de los países desarrollados. Ese fue el marco previo de intervención para organismos como el Banco Mundial (BM) para el financiamiento a la educación y la investigación, a partir de 1980. Así, BM, se convirtió en la principal agencia de financiamiento internacional a la educación de los países subdesarrollados mediante préstamos, pues la UNESCO se enfrascó en discusiones entre países recientemente independizados adscritos al bloque soviético y países occidentales (Tomasevski, 2004:134). Los préstamos del BM incluían becas para estudiantes universitarios del Tercer Mundo y cooperación técnica 
que se convirtió en el preludio de políticas educativas globales, e implicó una disminución del financiamiento para la educación superior -en la cual se incluía la ciencia y la tecnología-, ya que se privilegió la educación básica bajo el supuesto de que con ello se privilegia a los niños para que concurran a educación, de allí que se iniciara una crítica a la educación superior pública, la cual tendría que ser pagada igual que cualquier otro servicio público dado que da lugar a determinados costos, olvidando que se el financiamiento a la educación es mediante la recaudación fiscal (Tomasevski, 2004:157-158).

Desde 1999, en la Conferencia Mundial sobre Educación Superior, la UNESCO llamaba prestarse atención al progreso de los conocimientos mediante la investigación, lo cual exigía garantizar condiciones profesionales y financieras apropiadas. Sin embargo, al definir a la educación superior como un servicio público, indicaba que:

Si bien se requieren fuentes de financiación diversificadas, privadas y públicas, el apoyo público a la educación superior y a la investigación sigue siendo fundamental para conseguir que las misiones educativas y sociales se cumplan de manera equilibrada. En la educación superior, la gestión y la financiación han de ser instrumentos de la mejora de la calidad y la pertinencia. Esto requiere la creación de capacidades y la elaboración de estrategias apropiadas de planificación y análisis de las políticas, basadas en la asociación entre las instituciones de educación superior y las correspondientes autoridades. Las instituciones han de gozar de autonomía en sus asuntos internos, pero han de rendir cuentas a la sociedad de modo claro y transparente. (Organización de las Naciones Unidas para la Educación, la Ciencia y la Cultura-UNESCO, 1999:7)

Si en 1999, durante la Primera Conferencia Mundial sobre Educación Superior, se pensaba la investigación como un medio para conseguir los 
fines educativos, en la Segunda Conferencia Mundial sobre Educación Superior (2009) la investigación realizada por las instituciones de educación superior se concibe como "responsabilidad social de contribuir a reducir la brecha en materia de desarrollo mediante el aumento de la transferencia de conocimientos a través de las fronteras, en particular hacia los países en desarrollo" (UNESCO, 2009:5) en realidad no se habla de generación de conocimiento en los países subdesarrollados, sino de transferencia, de allí que se ponga atención a los mecanismos para incrementar la circulación de competencias de investigación, la creación de redes internacionales entre instituciones e intercambios de personal y alumnos, la convalidación de estudios para lograr crear capacidades nacionales y "garantizar la calidad y sostenibilidad de los sistemas de educación superior" (UNESCO, 2009:6) Asimismo alienta la participación entre sector público y sector privado para investigar e innovar, especialmente a las pequeñas y medianas empresas, lo cual quiere decir, que las patentes rentables se mantienen en manos de las grandes transnacionales, a la vez que se justifica el traspaso hacia el financiamiento privado -o al menos un aumento- en el conjunto de la educación superior debido que, según la Organización de las Naciones Unidas para la Educación, la Ciencia y la Cultura (UNESCO), "los fondos públicos son limitados y tal vez no resulten suficientes para un sector que evoluciona tan rápidamente" (UNESCO, 2009:9).

Por su parte, la Organización para la Cooperación y el Desarrollo Económicos (OCDE) consideraba que "la ciencia, la tecnología y la innovación... son factores decisivos para lograr un desarrollo social y económico sostenible" y lo que le preocupa es, por una parte la demora de la innovación ante la recesión de principios del 2000 , a la vez que consigna la disminución de los recursos públicos en el gasto bruto en investigación y desarrollo, entre 2008 a 2012, debido a los problemas fiscales que enfrentan 
los gobiernos, mientras que la inversión empresarial disminuyó, según la OCDE tal situación se debe a una disminución de la demanda, lo cual se expresa en una reducción de patentes registradas relacionadas con activos físicos frente a "activos basados en el conocimiento" que supone uso de software, por ejemplo (Organización para la Cooperación y el Desarrollo Económicos, 2014, pp.7,9). Para la (OCDE) el desarrollo de ciencia, tecnología e innovación es la triada que permite aumentar el crecimiento económico y la competitividad como base para generar empleo, para crear modelos de desarrollo económico que permita transitar a un uso menor de carbono; para que se diseñen nuevas tecnologías para coadyuvar en el tratamiento de problemas de salud; para emplear las TIC para contribuir a reducir la pobreza al incluir a individuos y empresas en actividades de innovación; con ello se supone que el acceso a las TIC contribuye a evitar la exclusión (OCDE, 2014:11-12).

Después de enumerar los beneficios del desarrollo de la ciencia, la tecnología y la innovación, para el crecimiento económico y el bienestar social, la OCDE recomienda el diseño de "un «nuevo pacto» para la innovación, el cual da prioridad en la agenda de las políticas públicas, al tiempo que buscan impulsar la financiación privada de innovación e incrementar el efecto de la acción pública" (OCDE, 2014:13). Ese nuevo pacto consiste en implantar "programas públicos con miras a reducir los costos administrativos y de aplicación”, incluidos los costos de evaluación de los programas; el diseño de "ecosistemas nacionales de ciencia, tecnología e innovación" que aporten valor y creen empleos; reforzar "la capacidad y el componente internacional de sus sistemas educativos y de investigación" en los países de la OCDE; incluso apunta hacia un apoyo público a la investigación y desarrollo empresarial para generar emprendimientos innovadores (OCDE, 2014:14-15), mientras que para 
la investigación pública recomienda sea de excelencia y apertura, se mantenga en "áreas de interés público o en aquellas en que las empresas no se adapten o no se sienten motivadas para invertir" y tenga la finalidad "de responder a los avances socioeconómicos y políticos más amplios y afianzar la competitividad”, de allí que para la investigación pública recomiende nuevas formas de financiamiento que aumenten la eficacia de los proyectos e instituciones, además de que se oriente por la transferencia y comercialización de conocimientos, mediante alianzas con el sector privado, la protección de los resultados y la participación de los estudiantes en la comercialización (OCDE, 2014:16,18).

La Comisión Económica para América Latina y el Caribe (CEPAL), desde 1979, sostenía que la maquinaria financiera tenía que apoyar el desarrollo de ciencia y tecnología, pero mantiene la idea de que los países subdesarrollados carecen de los conocimientos necesarios para generar y asimilar procesos tecnológicos, ni tienen capacidad para el diseño e ingeniería necesarios para la innovación, de allí que suscriba la idea de que los países latinoamericanos tienen que desarrollar la capacidad para emplear tecnología local o importada y recibir capacitación científica, técnica y administrativa para usar racionalmente la ciencia y la tecnología (Comisión Económica para América Latina y el Caribe, 1979:78). Para la década de 1990, la CEPAL procede a enmarcar la "investigación dirigida al desarrollo tecnológico y a las ciencias básicas", en el contexto donde el Estado tenía que "complementar el esfuerzo privado en materia de reinserción internacional y minimizar el costo social del ajuste" (CEPAL, 1996:55) y pone atención a los indicadores en ciencia y tecnología tales como graduados universitarios y gastos en investigación y desarrollo, destacando la relación entre progreso técnico y competitividad vinculada a una transformación productiva, como simple relación entre "el 
comportamiento de los agentes empresariales, la estructura sectorial y la vinculación entre los mercados interno y externo"(CEPAL,1996:67), relación simple ya que no reflexiona respecto al modo en que dicha estructura productiva está dominada por capital externo que obtiene beneficios de la explotación de patentes monopolizadas. Se llega a afirmar que los "órganos de vinculación permiten que la investigación científica repercuta en la vida cotidiana... los científicos forman alianzas con el sector empresarial y con el gobierno". (Solano Flores et al., 2012:28). A partir de la vinculación investigación científica, empresas y gobierno se cree es posible la revolución tecnológica que lleve a "diversificar la economía de los países de la región aplicando políticas industriales activas en combinación con políticas macroeconómicas, sociales y laborales [que] permitirían dinamizar la estructura productiva, fortalecer a las economías ante choques externos y garantizar la protección social" (CEPAL-Naciones Unidas, 2012). De esta forma, el apoyo a la investigación científica y tecnológica se concibe en términos de creación de conocimiento innovador en el sentido promover nuevos modelos de negocios con base en el uso de internet, de manera que se contribuya a resolver los problemas económicos, sociales, de empleo y bienestar.

\section{Las políticas de financiamiento a la ciencia y tecnología en México}

A fines de la década de 1970, se elaboró el Programa Nacional de Ciencia y Tecnología (1978-1982), apoyado por CONACyT que construye el primer acercamiento de gestión y organización a la ciencia y tecnología, por parte de gobierno, instituciones de nivel superior e investigación y sector productivo que utilizados como estrategia de desarrollo científico y tecnológico daría paso a las prioridades en la política social y la economía. A partir de esta iniciativa se "analizan las teorías del desarrollo tecnológico de los países del tercer mundo, la discusión de los problemas de la 
dependencia tecnológica y trata en detalle las dificultades y avances del desarrollo del sistema científico y tecnológico de México en su conjunto y aspectos de este desarrollo, por sectores de actividad productiva y de recursos disponibles" (Programa Nacional de Ciencia y Tecnología, 1978:21).

Actualmente podemos ver que la plataforma para el Consejo Nacional de Ciencia y Tecnología (CONACyT), creado en 1970, "es un inventario minucioso de ofertas y necesidades planteadas por numerosas intuiciones y grupos de los especialistas más destacados del país" (Programa Nacional de Ciencia y Tecnología, 1978:22), que utilizó mecanismos (programas, comités, grupos y convenios de cooperación científica internacional) para derivar acciones y metas específicas de formación de recursos humanos que demanden sectores de investigación, educación, producción y administración pública mediante la asignación de becas que, preferentemente, fueron destinadas a instituciones con programas en marcha. Respecto al gasto nacional en ciencia y tecnología se indica que CONACyT "coordinará los diferentes sectores e instituciones donde se realizan programas y proyectos, definirá la política de formación de investigadores de alto nivel, técnicos y especialistas, y determinará el carácter de cooperación internacional" (Programa Nacional de Ciencia y Tecnología, 1978:49).

A partir de 1990, la Secretaría de Educación Pública (SEP) "estableció como política privilegiar la creación de instituciones públicas en los estados bajo el régimen de organismos descentralizados de los gobiernos estatales. Surgen así los institutos tecnológicos estatales con el mismo modelo educativo que los federales... con una estructura organizativa novedosa 
y un modelo educativo diferente" (Rubio Oca, 2006:93-94), así como las llamadas universidades públicas de apoyo solidario y las universidades tecnológicas cuyo rasgo común es que sus directores o rectores son designados por el gobernador de la entidad federativa donde se localicen o por el Secretario de Educación Pública. También, durante este periodo, se expide Ley para el Fomento de la Investigación Científica y Tecnológica (LFICyT) y en conjunto con el Programa Especial de Ciencia, Tecnología e Innovación, establecen el cumplimiento de los mecanismos estratégicos para mantener un flujo permanente que sustente la formulación de las actividades de fomento del desarrollo científico y tecnológico en el país. Por ello, el CONACyT organismo público descentralizado del Estado tiene la facultad de plantear y ejecutar políticas encaminadas a la investigación del gobierno federal y asume la iniciativa para lograr llevar a cabo la creación y el funcionamiento del Sistema de Centros Públicos de Investigación del CONACyT desligados de las instituciones de educación superior pública y establece la toma de decisiones en materia de investigación y solución de los grandes problemas nacionales y el aumento de los niveles de productividad, competitividad y promoción del desarrollo económico.

En 1971, se crea el Sistema de Centros Públicos de Investigación del CONACyT constituido por 27 instituciones, de los cuales 26 son centros de investigación que argumentan la necesidad de crear instituciones que respondieran al avance científico y tecnológico, que descentralizaran la investigación y formaran investigadores, profesores especializados $\mathrm{y}$ técnicos expertos que contribuyeran a resolver los problemas nacionales. Lo paradójico de la creación de los centros de investigación CONACyT es que son promovidos por profesores de la UNAM y el IPN, entre otros, que se desligan de dichas instituciones. El primero de esos centros se creó en 1971: el Instituto Nacional de Astrofísica, Óptica y Electrónica, 
indicando que desde ya existía el Observatorio de Astrofísica Nacional de Tonantzintla, Puebla, dependiente de la Secretaría de Educación Pública (Presidencia de la República de los Estados Unidos Mexicanos, 1971), entre las funciones que se le atribuyeron fueron:

la preparación de "investigadores, profesores especializados, expertos y técnicos en astrofísica, en óptica y en electrónica [para] procurar la solución de problemas científicos y tecnológicos, relacionados con las citadas disciplinas $[\mathrm{y}]$ orientar sus actividades de investigación y docencia hacia la superación de las condiciones y resolución de los problemas del país" además de brindar servicios y asesoría técnica a la industria de manufacturas y a las compañías de ingeniería $[y]$ comercializar las tecnologías desarrolladas y los resultados que se obtengan de las investigaciones (Presidencia de la República de los Estados Unidos Mexicanos, 1971, Cap. I, Art.3).

Cabe indicar que, los primeros centros de investigación CONACyT se constituyeron como organismos públicos descentralizados, otros comenzaron como empresa de participación estatal como el caso del Centro de Investigación en Alimentación y Desarrollo, o bien, como asociaciones civiles y comparten un rasgo común: sus directores son designados por el Secretario de Educación Pública, tal como se establece en sus respectivos decretos de creación.

A partir de la Ley de Ciencia y Tecnología (LCyT) y con la participación del Sistema Nacional de Ciencia y Tecnología (CONACyT) se delinea una política de Estado que escinde la investigación científica de las instituciones de educación superior públicas, pues para estas se establecen programas específicos de formación de profesores con posgrado y su adscripción a cuerpos académicos que, más bien se atan a la asignación de recursos financieros extraordinarios consistentes en becas para posgrado $o$ financiamiento de proyectos de investigación. Así es como se ha entendido 
el gobierno federal al crear, a través de CONACyT, el Consejo General de Investigación Científica, Desarrollo Tecnológico e Innovación para "la articulación de políticas, la propuesta de programas prioritarios y áreas estratégicas, así como para la vinculación de la investigación con la educación, la innovación y el desarrollo tecnológico con los sectores productivos y de servicios." (Cámara de Diputados del Honorable Congreso de la Unión, Ley de Ciencia y Tecnología, 2015, Cap. II, Art 8, p.6). Los centros de investigación de CONACyT se presentan como intuiciones que median entre las instituciones de educación superior públicas, la investigación y la vinculación con el sector productivo.

La política federal aporta los recursos para la operación de cada institución potenciando en la medida que las instituciones de educación superior se ciñan a las políticas prioritarias para fortalecer la educación superior tecnológica en los estados y regiones del país, y con ello contribuir a mejorar la pertinencia de la oferta educativa del sistema de educación superior en todo el territorio nacional. (Rubio Oca, 2006:103). El Gobierno Federal, hasta 2018, en acuerdo con los gobiernos estatales en el marco del Programa Nacional de Educación (PRONAE) tenían como propósitos: ampliar la cobertura con sustento en planes estatales de desarrollo de la educación superior y de la ciencia y la tecnología que comprendan estudios de oferta y demanda, proyecciones de crecimiento, uso óptimo de la capacidad instalada, situación del mercado laboral y las necesidades de formación de profesionales, científicos, humanistas y tecnólogos para coadyuvar al desarrollo sustentable de la región, procurando la inclusión de los grupos de población. (Rubio Oca, 2006:95). Mientras que en el gobierno encabezado por Andrés Manuel López Obrador se habla de una reestructuración del CONACyT y de los apoyos a la investigación, aunque se mantiene la idea de crear "nuevos 
Centros Públicos de Investigación en estados de la república que carecen hasta ahora de ellos, en resonancia con la satisfacción de necesidades locales" (Álvarez-Buylla Roces, 2018:3) lo que pretende ser distinto es la transparencia, la eficiencia y la austeridad y la orientación hacia lo público, lo comunitario, poniendo límites al interés privado.

En realidad, se mantiene la política de separar la investigación de las instituciones de educación superior públicas con el argumento de que se trata de descentralizar la investigación, cuando en los hechos se tiende a producir una separación de las instituciones de educación superior públicas de la investigación, al mismo tiempo que segmenta, diferencia a las instituciones de educación superior mediante el otorgamiento de financiamientos para la formación docente y de investigación. Se impone una racionalización de la educación superior centrada en el cumplimiento de criterios tales como el número de profesores con posgrado, especialmente con doctorado, el total de profesores de tiempo completo en cuerpos académicos, el número de profesores pertenecientes al Sistema Nacional de Investigadores y adscritos a programas de posgrado registrados en el Padrón del Programa Nacional de Posgrados de Calidad (PNPC), entre los indicadores más destacados, con la peculiaridad, de que la exigencia de organizar cuerpos académicos se impone a las universidades públicas estatales, institutos tecnológicos, mientras que tales formas de organización no se advierten en las universidades públicas federales o en los centros de investigación de CONACyT.

En 2002, la SEP y CONACYT establecen el PNPC que "reconoce ahora la buena calidad de los programas de posgrado en todas las áreas del conocimiento, así como todas sus orientaciones (a la práctica profesional o a la investigación) y perfiles de egreso de sus estudiantes"(Rubio Oca, 2006:149); por supuesto que los centros de investigación de CONACyT 
cumplen completamente con los requisitos, siguiendo las universidades federales como la UNAM, la Autónoma Metropolitana, El Colegio de México o el Instituto Politécnico Nacional, con dificultadas siguen las universidades públicas estatales autónomas y después están los institutos tecnológicos - tanto federales como descentralizados- las universidades públicas estatales de apoyo solidario pero que en realidad no tienen autonomía sino que se crearon como organismos públicos descentralizados de los gobiernos estatales, y ni se diga de las universidades tecnológicas y politécnicas o las escuelas normales que, aunque tienen la función de investigación su organización académico-administrativa y la orientación de sus programas de estudios tienen propósitos distintos a la investigación.

Por otro lado, en cuanto al sector empresarial el gobierno federal en materia científica "ha impulsado e incorporado las MiPyMEs en actividades de innovación a través del fondo sectorial constituido entre la Secretaría de Economía y el CONACyT, a partir de 2004. En este esquema se busca apoyar a esas empresas para que realicen desarrollo tecnológico de productos, procesos, materiales y servicios de valor agregado, ya sea de manera individual o en colaboración con IES o centros de investigación. También apoya a las empresas grandes que involucren en sus proyectos a las MiPyMEs" (Presidencia de la República de los Estados Unidos Mexicanos, 2008-2012:61). La vinculación de la investigación con el sector productivo se expresa, también en los 109 de investigadores del sistema nacional que trabajaban -en 2018- en empresas privadas tales como ASTRAZENECA, Bayer de México, Centro Médico ABC, Centro de Ingeniería Avanzada en Turbomáquinas, Intel Tecnología de México, MABE, Polioles, o Fundación Teletón México, entre las más conocidas. 


\section{El financiamiento a ciencia y tecnología en México}

La crisis económica de los ochenta, provocó el restablecimiento de una nueva relación entre Estado e instituciones educativas en el marco de una política ligada al financiamiento público. Si bien es cierto que un país necesita de un proyecto financiero para lograr la sostenibilidad de una nación, en México iniciar un proyecto científico-tecnológico implica considerar un sistema que gestiona recursos, donde a partir de ciertas particularidades económico-financieras, organización y cumplimiento de funciones específicas puede operar y desarrollarse para emprenderlo; por ello, el discurso propio de este siglo podría comenzar por considerar que "el éxito económico de un país depende también del desarrollo y su aseguramiento financiero; y de la creatividad de las personas, sobre todo, para generar, divulgar y aplicar nuevos conocimientos" (De Almeida \& Arrechavaleta Guarton, 2017:4).

En países en vías de desarrollo se ha impuesto la idea de que para alcanzar altos niveles de vida debe prevalecer el modelo de producción de conocimientos y proyecciones de ciencia, tecnología e innovación como ocurre en los países con altos índices de desarrollo, por ello, cumplir con este tipo de prácticas atiende al beneficio social y, por ende, origina potencialidades financieras a escala nacional en Instituciones de Educación Superior e Instituciones de Investigación y Desarrollo. En 1970 el financiamiento en ciencia y tecnología en universidades "dependía del gobierno federal y estatal, quienes se responsabilizaban de cuatro quintas partes del mismo, con lo que quedaba un $20 \%$ a cargo de los ingresos propios de las universidades. Sin embargo, una década después la dependencia financiera de las IES del subsidio público, particularmente federal, se hizo casi total. (Casas \& Luna, 1997:77-78), lo que anularía su completa participación y aportación financiera. Para 1990, se establece 
y se desarrolla la política de modernización que causa como efecto la diversificación del financiamiento en IES y las relaciones entre industria y sociedad. La postura demandante de instituciones como la SEP y ANUIES, tuvo como finalidad abrir una brecha hacia la diversificación de las fuentes de financiamiento en instituciones de educación superior y su vinculación con el sector social y productivo. Para lograr una mayor eficiencia de las universidades y embonar conjuntamente con este sistema mexicano se requieren "fondos gubernamentales que deben distribuirse entre instituciones públicas y privadas de tal manera que se incremente al máximo la combinación deseada de calidad y cantidad" (Aboites, 1997:248).

Labra Manjarrez, propone como metas "incrementar anualmente los recursos de los programas gubernamentales orientados a ampliar la oferta educativa y el fomento a la mejora de la calidad de la educación superior y su aseguramiento en programas y fondos, tales como: el Programa para el Mejoramiento del Profesorado(PROMEP); el Fondo para la Modernización de la Educación Superior (FOMES); el Programa de Apoyo al Desarrollo Universitario (PROADU); el Fondo de Aportaciones Múltiples (FAM), el Fondo de Inversión para Universidades Públicas con Programas Evaluados (FIUPE), etcétera". (Labra, 2006:107). El elemento central para impulsar este sector, es la formación de recursos humanos, donde sobresale la promoción a los estudios de posgrado y la consolidación del Sistema Nacional de Investigadores (SNI). Para otorgar financiamiento a la educación y su instrumentación se estableció un conjunto de estrategias tales como: el "Programa de Becas para Estudios de Posgrado, a través de CONACyT, el más importante de México y reconocido a nivel nacional e internacional, brinda acceso a la población para realizar estudios de alto nivel en instituciones académicas de excelencia, tanto en el país como 
en el extranjero. A través de las distintas modalidades del programa, se otorgan becas para realizar estudios de posgrado a nivel de maestría, doctorado y posdoctorado, además de apoyar diversas especialidades técnicas y académicas". (CONACYT, 2019). Apoyado de la Secretaría de Educación Pública, esta institución "establecerá los mecanismos de coordinación y colaboración necesarios para apoyar conjuntamente los estudios de posgrado... en todas las áreas del conocimiento y el desarrollo tecnológico". (Cámara de Diputados del Honorable Congreso de la Unión, Ley de Ciencia y Tecnología, 2015, Cap. VIII, Art 42, p.26).

A partir del 2015 las becas de posgrado tienen un alcance sobresaliente en cuanto al recurso destinado -como se muestra en la Gráfica 1-, pues mantuvieron un crecimiento y una constante durante los siguientes años; mientras que los proyectos de investigación registrados en 2000 se mantuvieron en cuanto al recurso público designado a estos; pero, no es hasta 2019 que sufre un pequeño declive. Por último, al personal dedicado a las actividades científicas y tecnológicas en 2010 alcanzaron los 40 millones de pesos, siendo este uno de los años en que más recibió financiación de carácter público.

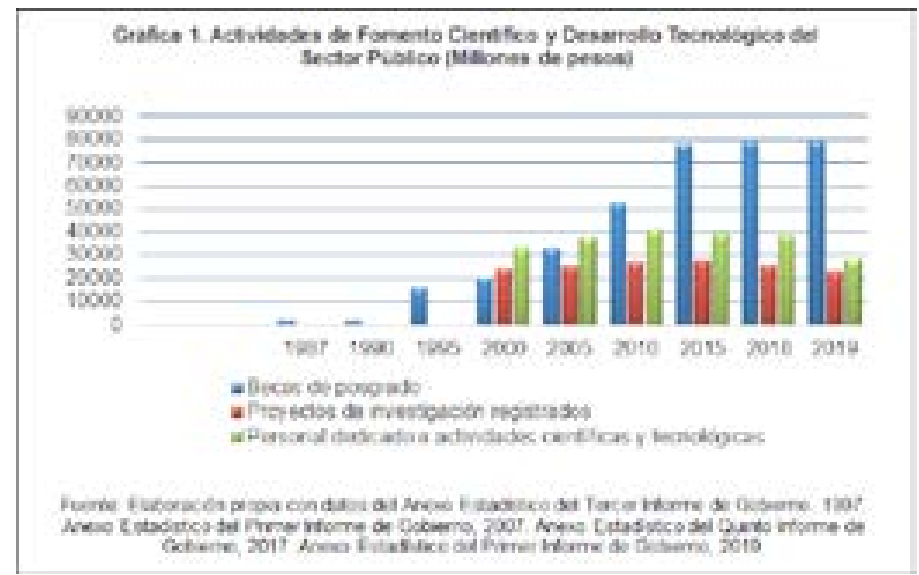


La operación y organización del Programa Nacional de Posgrados de Calidad tiene varias vertientes tales como la profesional, industria e investigación, así tomando en cuenta la cultura de evaluación en todos los ámbitos académicos y la asignación de fondos a través del concurso y competencia este programa funciona a partir de la siguiente clasificación por niveles:

“Competencia Internacional: programas que tienen colaboraciones en el ámbito internacional a través de convenios; incluyen la movilidad de estudiantes y profesores, la codirección de tesis y proyectos de investigación conjuntos. Consolidados: programas que tienen reconocimiento nacional por la pertinencia e impacto en la formación de recursos humanos de alto nivel, en la productividad académica y en la colaboración con otros sectores de la sociedad. En Desarrollo: programas con una prospección académica positiva sustentada en su plan de mejora y en las metas factibles de alcanzar en el mediano plazo y por último de Reciente Creación: programas que satisfacen los criterios y estándares básicos del marco de referencia del Programa Nacional de Posgrados de Calidad" (Consejo Nacional de

Ciencia y Tecnología, 2019).

El recurso monetario destinado por CONACyT en actividades de fomento científico y desarrollo tecnológico y específicamente en becas de posgrado, se distribuyen en becas nacionales y al extranjero. Sin embargo, los recursos para las becas para posgrado van en declive, como se ilustra en la Gráfica 2, donde se advierte la tendencia a disminuir, especialmente las becas al extranjero; pues, solo en el 2015 se alcanza a percibir un pequeño aumento del recurso destinado a estas becas, pero aun así no es realmente sobresaliente. Sin embargo, las becas de posgrado nacionales reflejan un considerable aumento manteniéndose hasta 2019. De hecho, aquí es notoria la consideración que el posgrado es el lugar privilegiado, no sólo para formar investigadores, sino el espacio de realización de investigación. 
Cratica 2. Actwidades de Fomenbs Dientifico y Desarrello Teenolegien CONAGy TBecas por Desfino [Malones de pesca)

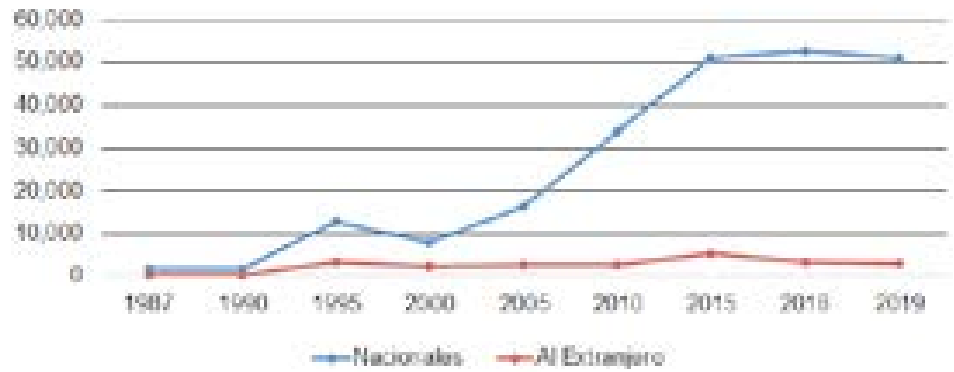

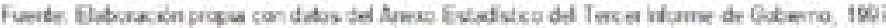

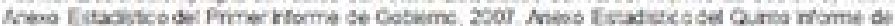

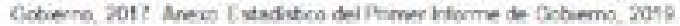

Así los beneficios que se logran adquirir a partir de esta participación son: reconocimiento de instituciones dentro de un padrón de CONACYT y de recursos destinados a la investigación y ciencia. La iniciativa de llevar a México a su máximo potencial aumento y por ello en el Plan Nacional de Desarrollo (2013-2018) se planteó el enfoque de una educación de calidad; de ahí, que por recomendaciones de la UNESCO se retomó la participación activa de un sistema de profesionalización docente con las capacidades de investigación-docencia típicas de la educación superior en el mundo; conocido como: el Programa de Mejoramiento del Profesorado (PROMEP) creado a finales de 1996, cuya meta es "buscar la integración y el desarrollo de cuerpos académicos que generen investigaciones de impacto regional y/o nacional que permitan mayor calidad en el profesorado para beneficio de los alumnos y la sociedad". (Secretaría de Educación Pública, 2016). Sin embargo, dicho programa para imponer una diferenciación salarial entre los profesores, pues quienes tienen acceso a las becas para posgrado y a organizarse en cuerpos académicos son los profesores de tiempo completo, lo cual excluye a los profesores contratados por horas o a tiempo parcial. Esa diferenciación entre el profesorado de educación 
superior se profundizó aún más, en 1984, al crear el Sistema Nacional de Investigadores (SNI), bajo el supuesto de que constituye un reconocimiento a quienes están dedicados a producir y colaborar en el conocimiento científico, además de coadyuvar a la descentralización de la ciencia, posibilitar la colaboración y coordinación intra e interinstitucional, así, como la relación entre investigación-docencia. Sin embargo, los resultados han sido aislar a los profesores pertenecientes al sistema nacional de investigadores en los posgrados, al mismo tiempo que se crean diferencias en los ingresos entre el profesorado de educación superior por la vía de los estímulos económicos mientras los salarios base se han modificado muy lentamente, así como la creación de una amplia base de profesores de tiempo completo incorporado en Cuerpos Académicos, donde, se supone se realiza investigación, como de modo individual sino como colectivo.

A pesar de que se insiste en que la investigación científica y tecnológica tiene su espacio privilegiado en los posgrados de las instituciones de educación superior, se ha creado un sistema de élite paralelo con la creación de los centros de investigación de CONACyT, así se escinde la investigación de las instituciones de educación superior públicas, con el agrupamiento de los profesores SNI en los posgrados, con lo cual la investigación se divorcia de los programas de formación profesional, a pesar de que se quiera destacar como una bondad que a partir de esa forma de organización "los investigadores que reciben financiamiento público adquirieron el derecho de explotar de modo comercial sus descubrimientos. En un mercado que se nutre de la innovación, esta circunstancia ha promovido que el sistema económico se base cada vez más en la generación de conocimiento y que las universidades sean un foco de innovación en constante diálogo con los sectores empresarial y gubernamental". (Solano Flores et al., 2012:29). Empero, no son las universidades públicas las que comercializan sus 
investigaciones, sino que ello se ha desplegado a través de los centros de investigación CONACyT.

A partir de 1978, se extienden las becas por nivel de estudio, en 1995 el capital destinado para estudios de maestría casi alcanza los 12 millones, a partir del 2005 logra impulsarse y comienza aumentar su recurso hasta alcanzar los 34 millones en 2015, en cambio, para estudios de doctorado, a partir de 2005 comienza ascender considerablemente y es hasta 2015 y cuatro años después que se mantiene en parámetros que alcanzan los 20 millones, se puede observar que los recursos destinados para becas de doctorado se redujeron, y se asocia a un limitación en el financiamiento de proyectos de ciencia (ver Gráfica 3).

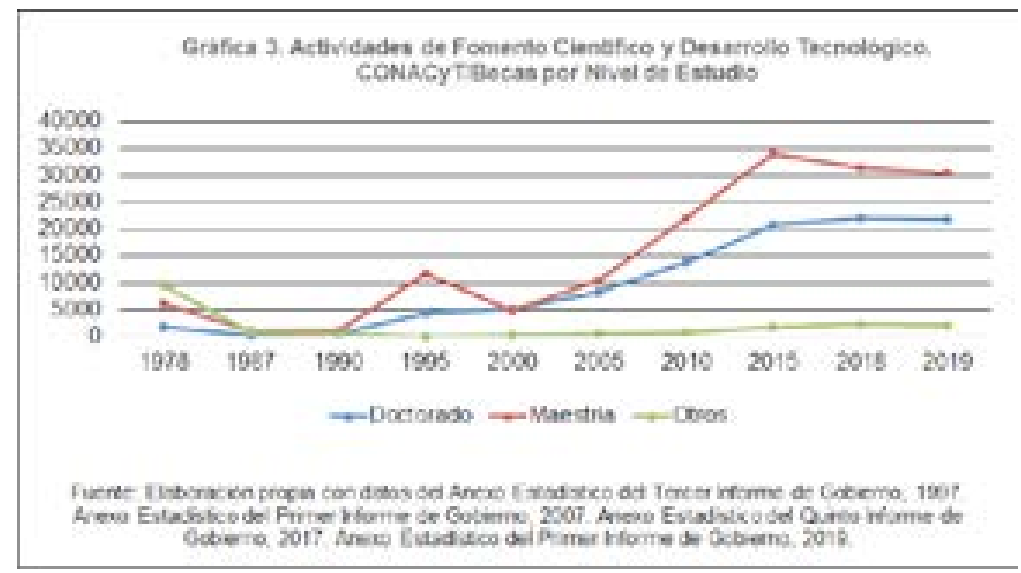

En diversos estudios acerca del financiamiento a la investigación se destaca que: no lograr asignar recursos suficientes a la ciencia; según el Programa Especial de Ciencia y Tecnología (PECYT) declara que no existe una estructura presupuestal nacional estratégica e integral que coordine el destino de la ciencia y tecnología y solo manifiesta distorsiones y limitaciones en el recurso, sin embargo, existe la "postura del Ejecutivo Federal respecto al financiamiento de la educación superior es contraria 
al mandato de la reforma al artículo 25 de la Ley General de Educación, aprobada por el Poder Legislativo en 2003, que establece la obligación de destinar recursos públicos a la educación equivalentes a $8 \%$ del producto interno bruto, de los cuales $1 \%$ corresponderían a educación superior" (Labra Manjarrez, 2006:120) y la a actuación de Ley de Ciencia y Tecnología en cuanto a lo que corresponde a este campo dispone que: "el Ejecutivo Federal y el Gobierno de cada Entidad Federativa, con sujeción a las disposiciones de ingresos y gasto público correspondientes que resulten aplicables, concurrirán al financiamiento de la investigación científica y desarrollo tecnológico. El monto anual que el EstadoFederación, entidades federativas y municipios destinen a las actividades de investigación científica y desarrollo tecnológico, deberá ser tal que el gasto nacional en este rubro no podrá ser menor al $1 \%$ del producto interno bruto del país mediante los apoyos, mecanismos e instrumentos". (Cámara de Diputados del Honorable Congreso de la Unión, LCyT, 2015, Cap. II, art 9 BIS, p.7). Entre los años 1990-2005 a diferencia de lo que se pudo obtener para la educación a nivel superior la participación del programa especial de ciencia y tecnología "incluyó como propósitos de la nueva política científica y tecnológica: avanzar en la descentralización de las actividades científicas y tecnológicas que se realizan en el país; impulsar una cultura nacional en la materia; elevar la competitividad e innovación en las empresas privadas y garantizar recursos públicos crecientes destinados a la investigación y desarrollo tecnológico". (Labra Manjarrez, 2006:124). Para impulsar y aumentar los niveles de productividad y competitividad en México se presentan datos del Gasto Federal ejercido en ciencia y tecnología por sector administrativo y el gasto en investigación científica y desarrollo experimental por ciencia. A partir de 1987, se muestra el comportamiento del gasto federal ejercido en ciencia y tecnología por sector administrativo, a mediados de la siguiente década la Secretaría de 
Educación Pública (SEP) y las paraestatales son las que hacen presencia de mayor gasto federal destinado a estas; pero no es hasta 2010 donde las paraestatales (empresas, organizaciones e instituciones) se desploman hasta estar por arriba de la media dejando muy por debajo a los centros públicos CONACyT; a partir de ahí los siguientes ocho años muestra un incremento en el gasto ejercido a pasos acelerados, para 2019 no muestra ninguna participación activa dentro del campo científico. En 2015, aumentó el gasto federal en CONACyT aun así no logró alcanzar la media. Actualmente el gasto federal ejercido en la SEP está por arriba de los 60 millones de pesos en ciencia y tecnología como se muestra en la Gráfica 4.

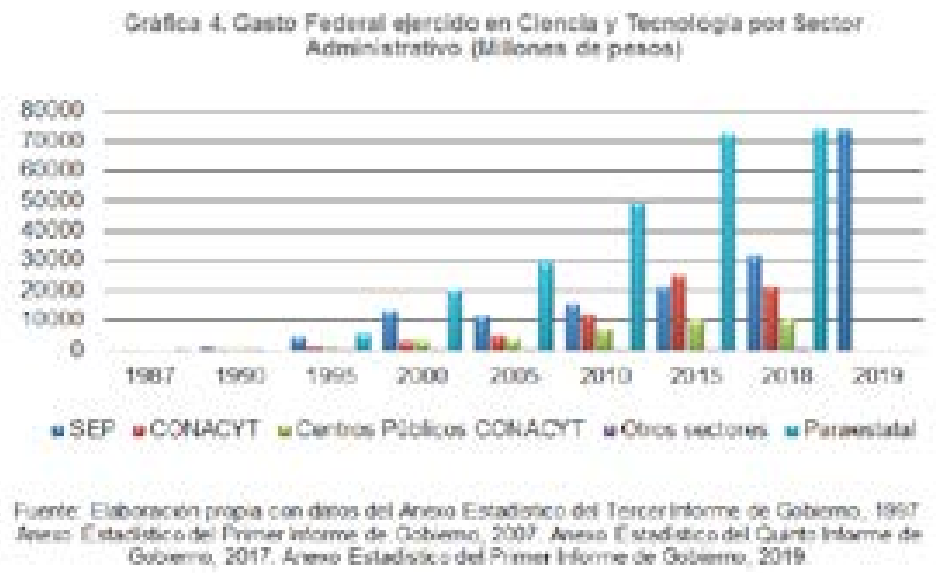

\section{RESULTADOS Y CONCLUSIONES}

A partir de examinar y analizar la postura de organismos internacionales y las políticas nacionales para el financiamiento a la ciencia y tecnología en México se encuentran los siguientes hallazgos o resultados, son: para los organismos internacionales, desde la década de 1960, México y los países subdesarrollados no tenían condiciones para la investigación científica y se proponían políticas de transferencia de tecnología de los países 
desarrollados a hacia los países periféricos, lo cual llegaba bajo la forma de inversiones extranjeras directas (instalación de empresas transnacionales); de esa concepción se pasó a un pensamiento donde se considera que los países menos desarrollados no cuentan con las capacidades para realizar investigación y se propone la formación de investigadores, especialmente en el extranjero y mediante la organización de posgrados; finalmente se concibe que la investigación carece de organización -tanto a nivel de las instituciones de educación superior como en su relación con el sector productivo- de allí que se proponga la creación grupos y redes de investigación que desplieguen capacidades innovadoras para proponer nuevos modelos de negocios o resolver problemas vinculados al mejoramiento de la productividad. Así, los vaivenes en el financiamiento a la investigación científica y tecnológica ha llevado a separar, paulatinamente la investigación de los programas de formación profesional para recluirla exclusivamente en los posgrados; sin embargo, también ha llevado a crear una segmentación entre los profesores de tiempo completo de las instituciones de educación superior pública, así como la creación de un circuito de centros de investigación adscritos al CONACyT apartados de las universidades públicas, lo cual ha creado un mecanismo de competencia por los recursos destinados a la investigación científica y tecnológica.

Para concluir, se puede afirmar que "el creciente papel de la ciencia y la tecnología en las economías de la región latinoamericana requiere una inversión sustancial en la infraestructura científica y tecnológica y en el desarrollo de personal capacitado de alto nivel". (Aboites, 1997:273) como puntos clave en los que han insistido las políticas de promoción de la investigación. 
Las recomendaciones de los organismos financieros internacionales, aunque se presentan como dirigidas a fomentar la investigación, en los hechos han llevado a una reorganización de la educación superior pública creando una diferenciación entre instituciones de educación superior.

Se advierte que la evolución del financiamiento, a partir de 1980, registra disminuciones en becas para posgrado, al modificarse los apoyos para proyectos y, hasta los estímulos lo cual crea una mayor selectividad-recursos concursables- entre instituciones y el profesorado de educación superior. Es preciso destacar que el financiamiento dirigido al campo científico sigue dos vertientes: una queda atado al posgrado en las instituciones de educación superior públicas, se va alejando de los estudios profesionales $y$, por otra parte, se dirige a centros de investigación que dependen del CONACyT separados de las instituciones de educación superior públicas.

\section{REFERENCIAS BIBLIOGRÁFICAS}

I Álvarez-Buylla Roces, M. E. (junio, 2018). Plan de reestructuración estratégica del Conacyt para adecuarse al Proyecto de Nación (2018-2024) presentado por MORENA. México, pp.42. Recuperado el [11/02/2020] de http://www.smcf.org.mx/avisos/2018/plan-conacyt-ciencia-comprometida-con-lasociedad.pdf

I Cámara de Diputados del Honorable Congreso de la Unión. Ley De Ciencia y Tecnología (LCyT). México. Publicada en el Diario Oficial de la Federación el 5 de Junio de 2002. Ultima reforma publicada DOF 0812-2015. Recuperado el [28/08/2019] de http://www.diputados.gob.mx/LeyesBiblio/pdf/242_081215.pdf

$\checkmark$ Comisión de Ciencia y Tecnología para el Desarrollo-UNCTAD. Examen de medios para conmemorar en 1999 el $20^{\circ}$ aniversario de la conferencia de Viena sobre ciencia y tecnología para desarrollo y la visión del papel de las naciones unidas en materia de ciencia y tecnología para el desarrollo. Ginebra, 12 de mayo de 1997, pp. 27. Recuperado el [22/11/2019] de https://unctad.org/es/Docs/ecn16_97d7.sp.pdf

I Comisión Económica para América Latina y el Caribe-CEPAL. (1979, January). Science and technology in Latin América: regional diagnosis and action programme. Recuperado el [28/10/2019] de https:// repositorio.cepal.org/bitstream/handle/11362/29699/S7900013 en.pdf?sequence=9\&isAllowed=y 
I Comisión Económica para América Latina y el Caribe-CEPAL. (1996). Transformación productiva con equidad. La tarea prioritaria del desarrollo de América Latina y el Caribe en los años noventa. Santiago de Chile, pp.185. Recuperado el [19/11/2019] de https://repositorio.cepal.org/handle/11362/2102

I Comisión Económica para América Latina y el Caribe-CEPAL. (2012). La CEPAL propone una visión integrada del desarrollo para la región. (Comunicado de prensa). CEPAL-Naciones Unidas. Recuperado el [28/10/2019] de https://www.cepal.org/es/comunicados/la-cepal-propone-vision-integrada-desarrollola-region

I Consejo Nacional de Ciencia y Tecnología-CONACyT. (2019). Becas y Posgrados. Gobierno de México. Recuperado el [14/10/2019] de https://www.conacyt.gob.mx/index.php/becas-y-posgrados

I Consejo Nacional de Ciencia y Tecnología-CONACyT. (2019). Programa Nacional de Posgrados de Calidad. Gobierno de México. Recuperado el [23/11/2019] de https://www.conacyt.gob.mx/index.php/ becas-y-posgrados/programa-nacional-de-posgrados-de-calidad

I Consejo Nacional de Ciencia y Tecnología-CONACyT. (octubre, 1978). Programa Nacional de Ciencia y Tecnología 1978-1982. México, pp.243. Recuperado el [04/12/2019] de http://www.siicyt.gob.mx/index. php/normatividad/nacional/programa-especial-de-ciencia-tecnologia-e-innovacion-peciti/1978-programanacional-de-ciencia-y-tecnologia

I De Almeida, Ó. G. \& Arrechavaleta Guarton, N. Á. (2017). "El financiamiento, la ciencia, la tecnología e innovación y la educación superior en los países en vías de desarrollo", en Revista Cubana de Educación Superior, Vol. 36, Núm. 3. La Habana, Cuba. Recuperado el [22/08/2019] de http://scielo.sld.cu/scielo. php?script=sci_arttext\&pid=S0257-43142017000300001

I Labra Manjarrez, A. (enero-abril, 2006). "Financiamiento a la educación superior, la ciencia y la tecnología en México”, en Economía UNAM, Vol.3. Núm.7. México, UNAM. Recuperado el [22/08/22019] de http:// www.scielo.org.mx/scielo.php?script=sci_arttext\&pid=S1665-952X2006000100006

I Organización de las Naciones Unidas para la Educación, la Ciencia y la Cultura-UNESCO. Conferencia Mundial sobre Educación Superior en el Siglo XXI: visión y misión. París, UNESCO, 1999, pp.9. Recuperado el [22/12/2019] de https://unesdoc.unesco.org/ark:/48223/pf0000117022_spa

I Organización de las Naciones Unidas para la Educación, la Ciencia y la Cultura-UNESCO. Conferencia Mundial sobre Educación Superior. La nueva dinámica de la educación superior y la investigación para el cambio social y el desarrollo (comunicado). París, UNESCO, 2009, pp.10. Recuperado el [22/12/22019] de https://unesdoc.unesco.org/ark:/48223/pf0000183277_spa

I Organización para la Cooperación y el Desarrollo Económicos-OCDE. Perspectivas de la OCDE sobre ciencia, tecnología e industria 2014 (Versión abreviada). Informe Iberoamericano. OCDE, 2014, pp.97. Recuperado el [19/02/2020] de https://read.oecd-ilibrary.org/science-and-technology/perspectivas-de-laocde-sobre-ciencia-tecnologia-e-industria-2014-version-abreviada 9789264226487-es\#page3

$\checkmark$ Presidencia de la República de los Estados Unidos Mexicanos. (2008-2012). Consejo Nacional de Ciencia y Tecnología, Decreto por el que se aprueba el Programa Especial de Ciencia y Tecnología 2008-2012, en DOF 16/12/2008. Primera Sección, pp.123. Recuperado el [28/1/2020] de https://www.conacyt.gob.mx/ cibiogem/index.php/programa-especial-de-ciencia-y-tecnologia-e-innovacion-2008-2012 
I Presidencia de la República de los Estados Unidos Mexicanos. (1971). Decreto por el que se crea el Instituto Nacional de Astrofisica, Óptica y Electrónica como organismo descentralizado de interés público, con personalidad jurídica y patrimonio propio, en DOF 12/11/1971. Recuperado el [19/02/2020] de https://www.inaoep.mx/ jgob/hjg/Facultades/Creacion.pdf

I Presidencia de la República. (1997). Anexo Estadístico del Tercer Informe de Gobierno. Gobierno de los Estados Unidos Mexicanos, México. Recuperado el [20/11/2018] de http://tercer.informe.zedillo. presidencia.gob.mx.

I Presidencia de la República. (2007). Anexo Estadístico del Primer Informe de Gobierno. Gobierno de los Estados Unidos Mexicanos. México. Recuperado el [14/08/2018] de http://primer.informe.calderon. presidencia.gob.mx.

I Presidencia de la República. (2017). Anexo Estadístico del Quinto Informe de Gobierno. Gobierno de los Estados Unidos Mexicanos. México. Recuperado el [23/03/2020] de https://framework-gb.cdn.gob.mx/ quintoinforme/5IG_ANEXO_FINAL_TGM_250818.pdf

I Presidencia de la República. 2019. Anexo Estadístico del Primer Informe de Gobierno. Gobierno de los Estados Unidos Mexicanos. México. Recuperado el [23/03/2020] de https://presidente.gob.mx/wpcontent/uploads/2019/09/PRIMER-INFORME-DE-GOBIERNO-2018-2019.pdf

I Secretaría de Educación Pública. (2016, 28 de diciembre). Novena Sección Poder Ejecutivo. Acuerdo número 21/12/16 por el que se emiten las Reglas de Operación del Programa para el Desarrollo Profesional Docente para el Ejercicio Fiscal 2017, publicado en DOF 28/12/2016. Recuperado el [29/11/2019] de https://www.dgespe.sep.gob.mx/public/ddi/prodep/Reglas_de_Operacion_PRODEP_2017.pdf

I Solano Flores, E., Martínez Martínez, E., Zaragoza, M. L., Figueroa Ramírez, B., Zúñiga Bello, P. y Juan P. L. (mayo-junio, 2012). "El financiamiento de la ciencia, la tecnología y la innovación: ¿es sólo cuestión de más dinero?", en Revista de Comercio Exterior, vol. 140. Núm. 6. México. Recuperado el [22/08/22019] de http://revistas.bancomext.gob.mx/rce/magazines/140/6/El_financiamiento.pdf

I Tomasevski, K. (2004). El asalto a la educación. Barcelona, Intermón Oxfam.

\section{BIBLIOGRAFÍA}

I Aboites, H. (1997). Viento del Norte: TLC y privatización de la educación superior en México. México.

I Casas Guerrero, R. \& Luna, M. (Coord.). (1997). Gobierno, academia y empresas en México: hacia una nueva configuración de relaciones. México, Plaza y Valdés.

I Rubio Oca, J. (2006). La política Educativa y la Educación Superior en México 1995-2006: Un Balance. México. Primera Edición. Fondo de Cultura Económica (FCE)_Secretaría de Educación Pública (SEP). pp.315. 
Envío a dictamen: 8 de mayo de 2020

Reenvío: 26 de mayo de 2020 Aprobación: 26 de junio de 2020

Heidi Mariana Archundia Esquivel. Licenciatura en Educación por la Universidad Autónoma del Estado de México, Facultad de Ciencias de la Conducta. Asesora educativa en distintas Instituciones de nivel superior. Asesora en planeación y organización cultural- educativo en el Departamento de Fomento y Patrimonio Cultural y Departamento Editorial en Metepec, Estado de México. 